\title{
La teoría de la traducción y la psicología experimental de los procesos de percepción del lenguaje *
}

\section{Mariano Garcia-Landa}

\section{INTRODUCCION}

Nuestra visión del lenguaje está dominada todavía por el texto saussuriano, que lo ve como el conjunto de la lengua («langue») y del habla («parole»). Pero ocurre que el texto saussuriano da la prioridad a la "langue» (sistema de signos), la cual, según ese texto, es lo único que puede ser objeto de ciencia. Para ese texto el habla es sólo el acto individual de fonación con el que los individuos «estropean" la regularidad gramatical de la "lengua». En cuanto tal acto individual, el habla no podría ser objeto de ciencia. La praxis de la traducción demuestra que el habla (oral y escrita) es la manifestación social esencial del llamado «lenguaje», que puede ser objeto de una ciencia que, desde luego, no será la lingüística, sino más bien la psicología.

\section{LA CIENCIA DE LA TRADUCCION}

El traducir, como el hablar, es una vieja actividad social de la especie bomo sapiens. Traducir consiste ni más ni menos que en hablar para redecir lo ya dicho por otro, por oral o por escrito, en un primer acto de habla. Ahora bien, esa frase que explica la traducción diciendo que traducir es, simplemente, hablar para redecir lo ya dicho, no ha sido posible hasta bien entrado el siglo XX, porque sólo en ese momento se pudo disponer del marco conceptual necesario para hacer la diferencia entre repetir palabras.

La explicación ingenua de la traducción pretendía que traducir consistía en repetir palabras, es decir, signos lingüísticos, de una lengua a otra; es decir, sin salirse de la «lengua» o sistema de signos, sin salirse, pues, de "lo lingüístico». La experien-

* Traducción con importantes modificaciones y adaptaciones del artículo del mismo autor "La theorie du sens", publicado por la Revue de l'Université d'Ottawa, vol. Is, núm. 3, julio-septiembre de 1981. 
cia de la traducción, por el contrario, es que el traductor "comprende» lo que alguien quiere decir realizando una percepción del lenguaje y, luego, una vez que esa percepción ha producido un percepto, habla para comunicar ese percepto. Seleskovitch (1968), Steiner (1975) y Garcia Yebra (1982) expresan esa idea diciendo que la actividad traductora tiene dos fases: la comprensión y la reexpresión. La ciencia de la traducción o traductología comienza, pues, proponiendo modelos de la percepción o comprensión del lenguaje.

\section{LOS MODELOS DE LA TRADUCCION}

Aunque el traducir, como el hablar, sea una actividad milenaria, sólo desde hace unos treinta años se comienza a constituir una ciencia de la traducción. Koller (1979) distingue la ciencia y la teoría de la traducción, considerando a ésta como la primera piedra del edificio traductológico, ya que su misión es definir lo que es la traducción, constituyendo así el campo de investigación de esa ciencia, es decir, su objeto. Entre los modelos propuestos figuran los de Nida, el de Richards (basado en el esquema de comunicación de Shannon y Weaver), así como los de Jumpelt, Houziaux y Bonnerot (Kelly, 1979). Desde Shannon y Weaver, casi todos los modelos abandonan el terreno de la lingüística para situarse en el terreno de la comunicación, y la prueba es que contienen tres agentes: r), un emisor de señales, que es el orador del discurso o el autor del texto; ambos, sujetos hablantes que «quieren decir algo»; 2), un receptor de señales, que es el interlocutor o sujeto de la percepción, y 3), entre ambos, un intermediario, el traductor, que realiza ambas operaciones, comprendiendo primero y hablando después, es decir, funcionando primero como sujeto de la comprensión y luego como sujeto de la producción de lenguaje.

\section{EL ACTO DE HABLA}

Esto equivale a decir que el concepto de acto de habla es esencial en toda teoría de la traducción... y también en toda teoria del habla, porque no hay diferencia entre ambas. Steiner (1979) sitúa claramente el problema: "Toda comunicación entre emisor y receptor, incluso en la lengua materna, ba sido reconocida como análoga a las modalidades de transferencia de sentido entre lenguas... No podrá baber una auténtica "teoria de la traducción" basta que no baya una teoria satisfactoria de cómo la mente bumana produce babla..., tal teoria de la traducción seria un modelo operativo de cómo se genera, percibe y memoriza el babla bumana.» Estas líneas describen el programa de la teoría de la traducción: construir un modelo del acto social de comunicación mediante un sistema de signos, en el curso del cual los individuos producen y comprenden habla, que es lo que nosotros llamamos telegráficamente «acto de habla».

Conviene no confundir nuestra definición y concepción del acto de habla como acto social de comunicación (producciónpercepción) con el speech act de Austin y Searle. Estos dos autores son filósofos especulativos que sólo se interesan por la lógica de las proposiciones emitidas o enunciadas. Nuestro modelo tiene una orientación experimental y se interesa, sobre todo, por la fase de la percepción, fase que no existe en el speech act de Austin y Searle.

Nuestro modelo pretende explicar los siguientes aspectos:

I. ¿Cuál es la diferencia entre la significación semántica (meaning, Bedeutung) de las palabras, tal como figura en los diccionarios y en esa abstracción que es el sistema semántico de la lengua, y lo que los habladores quieren decir, que no figura en ningún diccionario ni en ningún sistema, sino que se encuentra, como fenómeno psicológico o, si se prefiere, psicosocial, en la intención subjetiva de decir, que es lo que Benveniste (r 974) llama «el sentido intendido por el que quiere decir»? Es la diferencia entre el significado semántico y el "sentido" intendido por los hablantes. La ciencia llamada lingüística estudia los sistemas semánticos. de esa institución social que es la llamada «len- 
gua», pero está claro que para estudiar el fenómeno del sentido intendido por los hablantes hace falta una ciencia distinta.

2. ¿Cómo pueden los sujetos hablantes, que se supone que conocen de antemano el sistema semántico de la "lengua» que hablan, comprender el sentido intendido por la intencionalidad subjetivo-psicológica de sus interlocutores? La respuesta a esta pregunta es una teoría de la comprensión del lenguaje (no de las frases, pues, sino del sentido intendido).

3. ¿Cuál es el objeto de esa comprensión? La respuesta a esta pregunta es una teoría de la percepción lingüística.

\section{LA PERCEPCION DE FRASES}

Imagínese el lector que se encuentra en un laboratorio de psicolingüística experimental. El lector tiene un par de audífonos en la cabeza, por los que le llegan frases aisladas. Cada vez que el lector - que en este caso es un auditor-crea percibir una palabra que empiece con el fonema "b" tiene que apretar un botón. Un aparato mide el tiempo de reacción (RT) entre el momento objetivo en que se lanza tal fonema y el momento en que el auditor pulsa el botón. El RT se mide en milisegundos. Este experimento, realizado con un número estadísticamente suficiente de individuos, ha permitido demostrar que los RT son más largos cuando hay una frase subordinada dentro de una frase principal. Lo que sirve también para demostrar que el cerebro humano tiene una capacidad limitada, puesto que no puede hacer bien tres cosas simultáneamente: percibir una frase, reconocer el fonema «b» y ordenar a su mano que oprima un botón.

Este experimento (y comenzamos ya a criticarlo) no somete a prueba la «comprensión del habla", sino solamente -y ya es mucho- la percepción de frases aisladas, cosa que no ocurre nunca en la vida social del habla. Hay que distinguir, pues, entre percepción de frases aisladas y comprensión del habla.

El experimento esquemáticamente des- crito - y bautizado "phoneme monitoring) por sus autores, Foss y Swinney- ha sido criticado también por otros psicolingüistas experimentales que pretenden que «la percepción de una sílaba es más rápida que la percepción de su fonema inicial» (Levelt, 1978), lo que significaría que primero se percibe la sílaba y luego el fonema inicial, con lo que la medición de los RT es equívoca. Y significaría, además, que la percepción de fonemas iniciales o intermedios es una tarea artificial, en el sentido de que nadie hace eso en la vida corriente porque exige un análisis intelectual del material verbal percibido, retenido artificialmente en la memoria a corto plazo, para obedecer a los caprichos metodológicos del experimentador. Los autores citados, Foss y Swinney, se vieron obligados por la crítica a modificar una de sus hipótesis implícitas (revelando así la debilidad de las hipótesis de base) y admitieron posteriormente, como hipótesis, que se perciben primero las palabras, después las sílabas y sólo después los fonemas.

Con este ejemplo queriamos dar una idea de los métodos de trabajo de algunos psicolingüistas experimentales para revelar el crítico problema de las hipótesis de base. Porque aunque el experimento es la base de la ciencia, no sirve para mucho si el marco hipotético es insuficiente. Centenares de esos experimentos se han hecho - $-y$ se siguen haciendo-- en los últimos veinte años. Han sido analizados, clasificados y criticados por Levelt (1978). Todos estos experimentos se conciben para verificar una hipótesis explícita, pero ésta presupone hipótesis implícitas criticables sobre lo que es la llamada upercepción del lenguaje». A estas hipótesis implícitas sobre la "percepción del lenguaje» se les puede llamar tranquilamente teorias de la percepción del lenguaje. Levelt (1978) distingue tres grupos de esas teorías: las teorías lingüísticas, las conceptuales y las comunicativas.

\section{LAS TEORIAS LINGUISTICAS}

Las teorías o hipótesis implicitas llama- 
das «lingüísticas» pretenden que percibir (¿comprender?) una frase es captar la estructura gramatical de esa frase y. que, por tanto, el resultado de la percepción (¿comprensión?) de la frase es esta frase misma. Es cierto que, con Chomsky, hacen una distinción entre la «surface structure" de la frase o estructura fonomorfo-léxico-sintáctica, y la udeep structure» o estructura semántica subyacente (¿copresente?). Según esta distinción, percibir-comprender una frase puede consistir ya en construir una representación mental de ambas estructuras, ya de sólo la estructura semántica (lo que supondría que la estructura de superficie se descarta tras elaboración). Nuestra crítica es la siguiente: si este modelo de la percepción del lenguaje fuese correcto y el resultado de la percepción fuese la frase misma o su estructura semántica, la traducción sería imposible, puesto que el traductor produce una frase distinta y una estructura superficial y semántica necesariamente distintas, puesto que se construyen con otro sistema, lo que tendría que producir en su interlocutor una percepción distinta, imposibilitando la comunicación. Ahora bien, es un hecho pragmático diario que la traducción es posible, ergo el modelo en cuestión es falso. Es falso si pretende explicar la comunicación social mediante sistemas de signos, pero podría ser eficaz si sólo pretendiese explicar la mera percepción de frases aisladas en situación de laboratorio. Lo malo es que nunca se hace esa distinción entre percepción de frases aisladas en laboratorio y comprensión social del habla en la vida real. Y esta falta de claridad conceptual sobre las hipótesis de base es criticable.

\section{LAS TEORIAS CONCEPTUALES}

Las teorías o modelos llamados conceptuales «describen el resultado final de la comprensión ("understanding", ya no "perception") en términos no-lingüísticos» (Levelt, 1978). Es decir, el resultado del proceso ya no es una representación mental de la estructura superficial o semántica de la frase (que son objetos lingüísticos), sino algo muy distinto, que no tiene nada que ver con los objetos lingüísticos, sino que es más bien de índole «conceptual». Schank, citado por Levelt (1978), sostiene que el modelo del «understanding» del lenguaje (nosotros traducimos por "comprensión") debe explicar cómo una producción lingüistica (a linguistic input o signostructo emitido) se transforma en «estructura conceptual».

Según Levelt (1978), las teorías o modelos lingüísticos y conceptuales no tienen por qué ser incompatibles, puesto que toda comprensión tiene que comenzar por lo que él llama «inmediate linguistic awareness», expresión ambigua, puesto que lo de «inmediate» parece sugerir que no se trata de una conciencia clara y explícita de la estructura gramatical y/o semántica de las frases, mientras que lo de "awareness" parece apuntar, por el contrario, a esa conciencia. Es evidente que para comprender el sentido intendido por el interlocutor, yo tengo que percibir las frases que emite, y percibir las frases incluye una especie de conciencia o registro de sus estructuras superficial y semánticoprofunda, pero, por otro lado, no hay que olvidar que, tanto los niños como los analfabetos, "comprenden» perfectamente lo que se les dice aunque no puedan ser conscientes explícitamente de la gramática de las frases. ¿No habrá que postular que la percepción de las frases, en cuanto objetos lingüísticos, es un acto reflejo $\mathrm{y}$, por tanto, inconsciente, aunque conscientizable? Esta posibilidad está claramente apuntada en el mismo texto saussuriano. Pero si se admite esta posibilidad, ¿de qué se ocupa la conciencia explícita? ¿De qué somos conscientes al hablar? Nuestro modelo tiene una respuesta clara: al hablar somos, ante todo, conscientes de lo que el interlocutor nos quiere decir, del sentido intendido por su querer decir, aunque no seamos casi nunca - en la vida real- conscientes de la estructura superficial, ni siquiera de la estructura semántica, de las frases con que nos lo quiere transmitir.

Es necesario reconocer, sin embargo, que las teorias o modelos llamados «con- 
ceptuales» tienen el mérito de incluir en el proceso de la comprensión del habla lo que los anglosajones llaman el "context» y nosotros llamamos la «situación». Hay que hacer una distinción muy útil entre lo ya comprendido en los minutos anteriores - que es lo que nosotros llamamos "contexto"- y el conjunto de conocimientos aprioristicos y consabidos que la ceremonia social del acto de habla convoca en el sujeto de la comprensión -que es lo que nosotros llamamos la usituación»-, quedando entendido que esos conocimientos son, a la vez, necesarios para la comprensión y totalmente extralingüísticos..., salvo el conocimiento práctico de la lengua en que se esté hablando. Esto es un argumento contra las teorías o modelos (o hipótesis implícitas) de los «lingüísticos», según los cuales para percibir (o comprender) una frase basta $y$ sobra con conocer la lengua en cuestión, lo que los experimentos realizados, entre otros, por nosotros (García-Landa, 1978) demuestran que no es asi.

\section{LAS TEORIAS COMUNICATIVAS}

Las teorias dichas «comunicativas» ven en la comprensión del lenguaje una interacción social en la que un interlocutor trata de captar las intenciones de su interlocutor y viceversa, en función de una situación común de comunicación. De esta manera, más realista, tanto la producción como la comprensión del llamado lenguaje son integradas en la totalidad de un complicado proceso de interacción social, que es lo que nosotros, y no sólo nosotros, llamamos «acto social de habla». Levelt (I978) dice: «La teoría del acto de habla ("speech act theory") ha cautivado la imaginación de los psicólogos experimentales." ¡Ya era hora! También de los teóricos de la traducción, sobre todo si, como nosotros, se ocupan experimentalmente de la traducción oral simultánea, y por razones evidentes, ya que en la dicha traducción es evidente la enorme presencia del acto social de comunicación lingüística con la presencia social de varios individuos miembros de la especie y el consiguiente "descuido" de las construcciones lingüísticas y la consiguiente concentración en lo que el otro está queriendo decir con la cara, con los gestos, tanto o más que con las "palabras". "La comprensión es (...) el proceso mediante el cual la gente llega a la interpretación que el hablador queria que dieran a esa frase enunciada en ese contexto» (Clark, 1978). Ese uintended interpreted meaning» es lo que llamamos en la teoría de la traducción el «sentido intendido». Pero es evidente que mientras lo sigamos llamando "sentido" no saldremos de la terminología de la lingüística, con el grave riesgo de que la palabra "sentido" se interprete en el sentido de «significación semántica» o, lo mismo de grave, el sentido que tal palabra adquiere en tal contexto y que determina la equivalencia de traducción. El «sentido» de que habla nuestro modelo - como veremos- no es un fenómeno lingüístico, sino psicológico. Corresponde al percepto que produce la percepción sensorial. Se trata del efecto total que produce el acto de habla $y$, en cuanto tal, nosotros no vacilamos en sugerir que se le conciba más bien como un «espacio perceptual» que, en nuestra terminología, llamamos «espacio sigma» $(\Sigma)$.

\section{LA PRODUCCION Y LA COMPRENSION DEL LENGUAJE EN LA PERSPECTIVA DE LA PSICOLOGIA COGNITIVA}

Situamos ahora el contenido de estas tres teorias en el marco del «acto cognitivo» tal como lo concibe la psicología de la cognición, cuyo modelo (Dodd y White Jr., 1980) es al mismo tiempo «estructural» (compuesto por estructuras jerarquizadas), "procesal» (esas estructuras son la base de varias operaciones o procesos) y «funcional» (el procesador central CP ejecuta varios programas). Las estructuras del modelo son: I. Una memoria inmediata a muy corto plazo (unos tres segundos) que tiene captores sensoriales y una memoria también sensorial. 2. Una memoria a corto plazo, llamada «working memory" (memoria de trabajo) o "active 
memory", que es a la vez un espacio de trabajo y las operaciones que en ese espacio efectúa el CP o procesador central. 3. Una memoria a largo plazo, llamada "permanent memory", que contiene espacios conceptuales activables; es decir, todo tipo de conocimientos, entre ellos, el de la lengua o lenguas en cuestión. 4. El procesador central, CP, que toma decisiones y ejecuta programas y operaciones. 5. Un órgano productor de respuestas. Estas cinco estructuras están representadas en el modelo por rectángulos unidos entre sí por flechas que simbolizan los procesos, las operaciones y las funciones del sistema cognitivo.

\section{LAS ETAPAS DE LA PERCEPCION/COMPRENSION}

El modelo contiene la descripción de las etapas en que opera el sistema cognitivo concebido como un sistema informatizado de tratamiento electrónico de la información. Elaborado en un principio para la percepción sensorial, este modelo se aplica también a la producción y a la percepción/comprensión del lenguaje (sin que los autores indiquen si se trata de la percepción de palabras o frases aisladas en laboratorio o de la comprensión del habla real). En la percepción lingüística, las ondas sonoras portadoras de las formas acústicas de la lengua o sistema de signos son captadas por los captores sensoriales y transformadas en corriente eléctrica neuronal. Esos datos sensoriales duran en los captores auditivos unos doscientos cincuenta milisegundos, pero son acumulados y mantenidos en la memoria auditiva inmediata a muy corto plazo durante unos tres segundos. Durante este tiempo el sistema cognitivo puede proceder a la selección, agrupación y extracción de características lingüísticas. Esta operación -que necesita la activación en la memoria permanente de los conocimientos de la lengua o sistema de signos en cuestión- es realizada por los «detectores de características lingüísticas» y conduce al reconocimiento de fonemas, sílabas, morfemas, palabras, construcción sin- táctica, etc., y, finalmente, de la representación semántica, la cual, según este modelo, es la fase final de la percepción lingüística (quedando siempre la duda de si se trata sólo de la percepción de frases o si el modelo incluye también la comprensión del habla real). El modelo reconoce explicitamente que el sistema opera en dos direcciones: el reconocimiento sucesivo de datos lingüísticos, a medida que las ondas sonoras van entrando en el sistema, se efectúa de manera ascendente («bottom up»), pero también, en un momento dado, de manera descendente ("top-down» o "flow-down»), puesto que la memoria permanente puede intervenir para reconocer, por ejemplo, las palabras, cuando el sistema cognitivo, a medida que van entrando las ondas sonoras, activa los conocimientos apriorísticos de la lengua en cuestión. No se nos dice en qué momento el proceso cognitivo entra en la conciencia explícita; tal vez a partir de la entrada en la memoria a corto plazo en cuanto que ésta se define como umemoria de trabajo", operativa o activa. En todo caso, es de suponer que la representación semántica se produzca en la conciencia explícita.

\section{CRITICA DEL MODELO DE LA PSICOLOGIA COGNITIVA}

Nuestra teoría formula tres críticas de este modelo:

Primera critica: el modelo es incompleto inclusive desde el punto de vista de la propia psicología cognitiva, puesto que plantea y no resuelve, no sólo el problema de la diferencia entre percepción lingüística de frases y palabras y comprensión del habla real, sino también el problema de la transformación de la información contenida en las representaciones semánticas con objeto de su almacenamiento en la memoria permanente. Las representaciones semánticas -que, según modelo, son el último producto del proceso- no pueden entrar en memoria permanente tal como están, sino que tienen que transformarse. Esta es una afirmación que hacen los autores en otras partes de su obra 


\section{Estudios}

(Dodd y White Jr., I980). Se nos habla de «unidades cognitivas» como el elemento que se extrae de la memoria permanente. Ahora bien, no se nos dice si estas "unidades cognitivas" son "representaciones semánticas» u otra cosa. Es de suponer, sin embargo, que los autores piensen que son otra cosa, puesto que las llaman de otra manera. No dicen nunca que se extraigan de la memoria las representaciones semánticas, sino las «unidades cognitivas». Si esto es asi, de ello cabe deducir que las representaciones semánticas tienen que ser transformadas en unidades cognitivas para que puedan entrar y conservarse en la memoria permanente. Si esto fuera asi, las representaciones semánticas son inmediatamente transformadas en unidades cognitivas y estas unidades cognitivas son el verdadero resultado del proceso de percepción/comprensión del lenguaje. Estas unidades cognitivas, definidas como "espacios conceptuales» (es decir, en modo alguno «lingüísticos»), son definidas por el modelo Elinor como espacios que contienen información sobre el estado, el cambio de estado, la causalidad y las acciones, o, por el modelo ACT, como estructuras lógico-proposicionales.

\section{HUELLA MNESICA, EVANESCENCIA \\ DE LAS PALABRAS Y EFECTO DE JARVELLA}

Detengámonos un momento en esta nuestra crítica del modelo de la psicología cognitiva para reflexionar un instante sobre la expresión "entrada en memoria». Nuestra teoría postula que lo que hace mella y deja huella en la memoria activa no son los significados semánticos de las palabras (que son un objeto lingüístico), sino el sentido en cuanto objeto psicológico o percepto percibido cuando el interlocutor comprende to que quiere decir el otro. Este postulado es estratégicamente decisivo para la construcción de nuestro propio modelo. Pero es más que un postulado. Creemos que se le puede considerar como un hecho suficientemente demostrado experimentalmente. I se reconoce como hecho es que el "material verbal" no es retenido por la memoria, sino sólo por la memoria a muy corto plazo, es decir, no hace mella ni deja huella. Esto fue postulado por Seleskovitch (1968), basándose en la experiencia de los intérpretes de conferencia que trabajan en la modalidad llamada "consecutiva» (repiten todo un discurso, no simultáneamente, sino después de que ha terminado el orador, basándose en notas ideográficas - no taquigrafia- tomadas mientras el orador habla). Es un hecho conocido que el intérprete no repite en esos casos necesariamente las palabras, pero sí el "sentido». Si se le pregunta al intérprete después, no recordará probablemente ninguna de las palabras ni de las estructuras sintácticas, pero sí las ideas. Seleskovitch (1968) lo llama el «principio de la evanescencia de las palabras». Es lo que los psicolingüistas experimentales llaman el "efecto de Jarvella" (Jarvella effect).

Jarvella realizó, entre i 970 y 1972, una serie de experimentos que consistian en presentar a sujetos ciertos discursos o textos, y en interrumpir de sorpresa la escucha o la lectura para pedirles que repitieran lo que acaban de oír o leer. Resultado: la memoria verbal de la última frase o parte de frase era aún bastante exacta, pero no la de las frases precedentes. Levelt (1978) - de quien tomo estodice: «El material (verbal) anterior ya había sido interpretado y sólo el último período o la última frase estaba disponible aún en forma literal (verbatim), no interpretada.» La palabra «interpretación», tal como la emplea aquí Levelt, tiene el sentido de «transformación del material verbal en el espacio conceptual", que es lo que los traductores llaman "sentido" y nosotros llamamos uespacio perceptual Sigma» ( $\Sigma)$. De esto podemos sacar la conclusión de que el proceso de comprensión -que consiste en una «interpretación»- descarta el material verbal - lo que los lingüistas llaman la «lengua»-- en cuanto llega a la fase final en la que se produce el percepto $\Sigma$.

Hay que tener en cuenta también el ya 
clásico estudio de Schas (1967), quien presentó a sus sujetos narraciones que contenían frases en voz activa y pasiva, para luego repetir algunas de estas frases cambiando o no la voz. Los sujetos tenían que decir si el segundo enunciado habia salido así, ya en activa, ya en pasiva, en la lectura original. Si acertaban, se hablaba de «reconocimiento». Pues bien, el reconocimiento era casi perfecto para las últimas frases "percibidas», pero para las anteriores descendía alrededor del 60 por 100 . Levelt (1978) comenta: «La memoria literal o lingüística («verbatim memory") de una frase se pierde rápidamente».

El lector puede hacerse una idea del contenido de estos experimentos sin más que intentar contar a un amigo la novela, libro o artículo que haya leído con anterioridad.

El efecto de Jarvella, o principio de la evanescencia de las palabras, es muy importante porque permite trazar claramente la frontera que separa a la "lengua» del «habla». Es uno de los pilares teóricos de nuestro modelo del "acto de habla» (que es un modelo o teoría de la comprensión del habla real).

Segunda crítica: el modelo de la psicologia cognitiva no está completo porque presenta la comprensión como si pudiera tener lugar sin una producción anterior, y viceversa, presenta a la producción como desconectada de la comprensión.

En la primera parte del capítulo que consagran a la producción y a la comprensión "del lenguaje», Dodd y White Jr. (1980) exponen un modelo de la producción "lingüística», y en la segunda parte, separado del anterior, presentan su modelo de la comprensión, pero no presentan un modelo unico de la producción y la comprensión juntas. Desde el "Tratado del hombre», de Descartes, los filósofos y los psicólogos han construido muchos modelos para explicar cómo el individuo humano, aislado y solo frente a la naturaleza y sus objetivos naturales, percibe los objetos sensoriales o cuerpos sensibles. Esos modelos conllevan, en general, tres elementos:

I. El objeto físico, sensorial o cuerpo sensible que se trata de percibir.

2. Los medios de transmisión, luz, ondas sonoras, que transportan los "datos sensibles» desde el cuerpo sensible hasta los órganos periféricos en que esos «datos físico-sensibles» se transforman en "datos sensoriales".

3. El percepto producido por el sistema perceptor. Los filósofos, sobre todo en este siglo, han debatido largo y tendido para dilucidar si se perciben también los datos sensoriales ("sense data») o sola y directamente el cuerpo sensible u objeto de la percepción. En la pintura impresionista se tiene a veces la impresión de que se perciben las pequeñas pinceladas como datos sensoriales de luz en que el pintor descompone el objeto.

Pero no hay que olvidar que el sujeto de la percepción sensorial se encuentra siempre solo frente al objeto natural, detrás del cual no hay nadie (ninguna persona) que tenga la intención de transmitirle, como mensaje, el objeto de la percepción. En la percepción lingüística, por el contrario, cuando llegan a nuestros oídos los «datos sensibles» que son las formas u objetos lingüísticos, no estamos solos, sino frente a un individuo de la misma especie que emite esas formas sonoras con la intención de comunicarnos algo que no es precisamente las dichas formas sonoras lingüísticas. ¿Qué es lo que nos quieren transmitir o comunicar? Desde luego, no las palabras. Esta distinción, sencilla y evidente, no la hacen los lingüistas. Por otro lado, la situación de percepción de eso que nos quieren comunicar es eminentemente social y esto tiene que tener una influencia en el modelo. Por ello, es absurdo presentar el modelo de la comprensión del lenguaje separado del modelo de la producción. Ambos son dos submodelos de un modelo más vasto, el modelo de la "producción-comprensión del espacio $\Sigma$ ", que nosotros llamamos taquigráficamente «modelo del acto de habla». Precisamente porque separan esos 
dos submodelos, los teóricos de la psicologia cognitiva no ven lo esencial: el objeto de la comprensión, que no es un objeto lingüístico, sino un objeto psicológico que nosotros llamamos «espacio $\Sigma »$. Así como el objeto de la percepción sensorial es el cuerpo sensible y no los datos sensoriales, el objeto de la percepción/comprensión "del lenguaje» es el espacio $\Sigma$ y no los datos lingüísticos como son las estructuras superficiales y las mismas estructuras semánticas. De ahi que tengamos no poca reticencia en hablar de la percepción o comprensión «del lenguaje" porque no es el lenguaje lo que se percibe o comprende sino otra cosa.

Tercera critica: el proceso de la comprensión "del lenguaje» no termina con la representación semántica, sino con el sentido de una forma

Si se aisla la cọmprensión de la producción, se reduce el modelo de la upercepción lingüística» a un mero modelo de la percepción sensorial de ondas sonorolingüísticas. Para evitar este error, proponemos nosotros la teoría de la percepción de formas simbólicas, cuya esencia es lo siguiente: suponga el lector que rueda por una carretera a $120 \mathrm{Km} / \mathrm{h}$. y de pronto ve un círculo rojo sobre fondo blanco con la cifra 80 en negro en el centro. El lector decelera. Unos kilómetros más abajo un psicólogo le detiene y le pregunta: ¿de qué material está construido ese círculo, de metal, de plástico o de madera?, ¿cuál es el diámetro del círculo rojo exterior? El lector responderá probablemente que no ha tenido tiempo para fijarse en esos detalles, que, de todas maneras, no tienen importancia. Lo único importante es el sentido del símbolo. $Y$, sin embargo, para comprender el sentido de ese signo, -el sentido que tiene colocado al lado derecho de la carretera, no la semántica que tendria si se encontrase tirado en el suelo de un depósito municipal de signos de circulación o el que pudiera tener si apareciese en un sueño- es absolutamente necesario "percibir" sensorialmente el soporte material. Lo mismo ocurre con el habla. Hay alguien que nos quiere comunicar algo (un sentido o espacio perceptual) y utiliza un soporte material que es un signostructo o configuración organizada de signos de un sistema.

El lenguaje humano -que es un medio de producción y comunicación de espacios perceptuales de tipo $\Sigma$, no de tipo sensorial - utiliza también un conjunto de formas simbólicas, construidas con el material de los signos de la lengua. Salvo que ese conjunto es mucho más complicado y complejo que el sistema simbólico de la circulación. Para empezar, las formas simbólicas, y sus correspondientes «sentidos», de la comunicación "lenguajistica», no «lingüistica», humana son infinitos, aunque las posibilidades del sistema de signos sean finitas. $Y$ es cierto que este sistema de signos funciona como un código pero sólo en cuanto a la construcción de signostructos, no en cuanto a su interpretación. Los principios de la "percepción de formas simbólicas» son, sin embargo, los mismos. Es decir, en toda percepción de formas simbólicas hay dos fases: una, la percepción, en general reflejada, inconsciente, del soporte material (lengua), y, dos, la interpretación o comprensión del «sentido de la forma simbólica». El soporte material de las formas simbólicas del habla son la estructura superficial ("significante» de la frase) y la estructura semántica («significado" de la frase). Estos dos niveles estructurales están presentes en el sistema cognitivo durante algún momento del proceso de comprensión del habla, pero no necesariamente de manera consciente, sino como mero acto reflejo, inmediato, de reconocimiento de ese material. En este sentido debe interpretarse la «immediate linguistic awareness" de Levelt (1978). Hay, sin duda, una recepción, una entrada y un reconocimiento, pero normalmente es inconsciente. $\mathrm{Ni}$ tampoco está dicho en qué momento se produce ese reconocimiento. Puede producirse antes o después de la constitución del percepto final o «espacio perceptual $\Sigma$ ». Ahora bien, las formas simbólicas son artefactos culturales (creados por los hombres, no por la naturaleza) y por ello es posible bajar el umbral de la conciencia explícita y ser consciente del soporte material (ver- 
bal). Es posible detener el auto, descender, comprobar si el disco es metálico o plástico y cuáles son sus dimensiones. En el acto de habla esto significa analizar gramaticalmente una frase, o ser consciente de tal o cual palabra, o de si el interlocutor ha empleado una pasiva directa o refleja. Esto es más fácil hacerlo durante la lectura, o sea, en los actos de habla escrita. En los actos de habla oral vamos siempre en auto y a más de I $20 \mathrm{Km} / \mathrm{h}$., raras veces nos paramos a pensar en el material lingüístico-verbal que oímos o producimos. Sólo por deformación profesional los lingüistas en sus gabinetes y los psicolingüistas en sus laboratorios se detienen a examinar las infrastructuras lingüisticas de la comprensión del sentido.

\section{NUESTRO MODELO DEL ACTO DE HABLA}

Las tres críticas que acabamos de formular nos preparan el terreno para construir nuestro modelo del acto de habla. Vamos a formularlo primero de manera literaria, luego vamos a hablar de su base experimental $y$, finalmente, lo formularemos en notación simbólica.

\section{LA DESCRIPCION LITERARIA DEL MODELO}

El acto de habla es una interacción social regida por normas sociales en la que varias personas tratan de comunicarse ciertas percepciones especiales (que sólo pueden producirse en el acto de habla), utilizando un sistema especial y específico de signos y reglas (llamado vulgarmente «lengua»). El hecho de que los miembros de una sociedad dada utilicen un sistema de signos y reglas para comunicarse percepciones especiales ( $y$, con ello, para producir esas percepciones especiales, distintas de las sensoriales) se llama «lenguaje». El así definido «lenguaje» consiste en actos de habla y se manifiesta en actos sociales de habla. El habla es la manifestación fundamental del len- guaje. El habla es una actividad social, que consiste en la producción y comunicación de percepciones especiales. En la interacción social del habla intervienen varios actores que desempeñan alternativamente dos papeles fundamentales, el papel social de la producción de esas percepciones especiales, que nosotros llamaremos sigmáticas para distinguirlas claramente de las sensoriales, con objeto de comunicarlas a otros miembros de la sociedad. El segundo papel es el de la compresión sigmática (hasta ahora llama$\mathrm{da}$, con imprecisión, «lingüística». El adjetivo «lingüístico» es impreciso, equívoco y ambiguo porque parece que dice que lo que se comprende es la "lengua", o sea, el sistema de signos y reglas, lo que no es cierto; o bien puede interpretarse en el sentido de que lo que se comprende es el «lenguaje», lo que tampoco es cierto. Lo único que se comprende es la percepción sigmática que el interlocutor produce con objeto de comunicarla).

El concepto de comprensión sigmática es el concepto fundamental del modelo. Definimos la comprensión sigmática como una clase especial de percepción, que presupone una capacidad de percepción sensorial, pero, por encima de ésta, supone otras muchas cosas más. La comprensión sigmática consiste en percibir lo que el interlocutor quiere decir; es decir, consiste en llegar a producir un percepto sigmático que sea de alguna manera idéntico al percepto sigmático que el interlocutor que desempeña el papel de la producción quiere comunicar (quiere decir). La comprensión sigmática se produce solamente en el interior del acto social del habla y consiste en producir un percepto sigmático, para lo cual es necesario interpretar la frase emitida en función de:

a) La situación social del acto de habla, la cual comprende una serie de elementos extralingüísticos y extrasemánticos, como son: la mímica y gestualidad expresiva del interlocutor, el conocimiento pragmático de las normas sociales que rigen el acto social del habla, que se puede llamar también experiencia de la vida. 
b) Una serie de conocimientos intelectuales, no pragmáticos, sobre el tema de que se esté hablando, conocimientos, además, que tienen que ser los mismos que conoce también de antemano el interlocutor. Es decir, se trata de conocimientos compartidos o consabidos. Entre estos conocimientos figura el conocimiento del sistema y reglas que se esté usando.

c) Entre estos conocimientos intelectuales (expresables) figura todo lo que se haya comprendido ya durante el habla (contexto, en sentido estricto).

El modelo postula un esquema general del proceso de comprensión sigmática, según el cual, la llegada de los datos lingüísticos (la frase antes de ser elaborada) al sistema cognitivo del sujeto de la compresión desencadena un proceso complejo que procede, ante todo y en primer lugar, a constituir una hipótesis total del percepto sigmático a constituir. Con esta hipótesis, el sujeto de la comprensión procede a verificar hipótesis sobre la estructura lingüística de la frase, de las palabras, de los fonemas; los cuales son perfectamente ininteligibles sin esa primera hipótesis general del percepto. (Sin saber lo que el otro quiere decir, no podemos oír las palabras de la frase, hecho que pretendemos haber demostrado experimentalmente, como diremos más abajo.) El objeto de la comprensión es el percepto sigmático, que es lo que se percibe inmediatamente y sólo después se «perciben» con claridad los datos lingüísticos o de la lengua. El proceso de comprensión comporta así tres momentos: 1), una primera percepción sensorial de las ondas sonoras (o grafismos) del comienzo de un hablar, cuyo resultado bruto se mantiene en la memoria a muy corto plazo hasta que se produzca una primera hipótesis de percepto sigmático («sentido»); 2), la producción de este percepto en cuanto hipótesis; 3), la verificación posterior de esta hipótesis en los datos lingüísticos retenidos, en cuyo momento y sólo en este momento se puede hablar de «percepción de la frase». Este es el principio de la "primacía del sentido" ("sentido» significa aquí "percepto sigmá- ticon), que, en nuestra opinión, como veremos, ha sido suficientemente demostrado experimentalmente.

Pero para poder constituir la hipótesis de percepto sigmático, el sujeto de la comprensión no sólo utiliza los datos lingüísticos, sino, sobre todo, otros datos extralingüísticos (extrasemánticos) como son los mencionados en a) (gestualidad y saber pragmático de las normas sociales) y b) conocimientos intelectualizables sobre el tema, la lengua y el contexto o acumulación de perceptos sigmáticos transcurridos.

Para hacernos una idea de lo que significa el elemento a), o saber pragmático, consideremos este caso: si alguien me da la mano por la mañana en Helsinki y masculla unas palabras que no entiendo porque me falta el conocimiento del finlandés, comprendo que me quiere decir «buenos días» a causa de la "situación». La situación en este caso significa un saber pragmático del ritual social europeo. O bien: si en un atasco de circulación en una plaza parisina a la hora punta de las seis de la tarde, yo hago una maniobra que molesta al conductor que está a mi lado izquierdo y éste mueve los labios haciendo muecas y visajes de determinada manera, yo sé que me está insultando, aunque no oiga en absoluto las frases que está emitiendo porque, además del ruido ambiente, nos separan dos cristales de ventanillas. En ambos ejemplos se trata de un caso límite en el que la «lengua» queda eliminada - y con ella la semántica, es decir, los significados de los significantes - para demostrar la independencia y autonomía del fenómeno fundamental, que es el percepto sigmático (más abajo llamado «espacio perceptual sigma»).

\section{LA BASE EXPERIMENTAL DEL MODELO}

Nuestro modelo nace de nuestra experiencia profesional de la interpretación simultánea (traducción oral simultánea). Para nuestra tesis doctoral (García Landa, 
I978) hemos realizado una serie de experimentos in vivo de una de las muchas situaciones reales del habla, la situación llamada "conferencia internacional», en la que varios oradores tratan de comunicar, mediante varias lenguas, gracias a la intervención de los intérpretes que «repiten en la otra lengua lo que han comprendido en una primera lengua» (o bien, como nosotros decimos, precisando la dicción, que reproducen en un segundo acto de habla lo que han comprendido en un primer acto de habla). La traducción oral simultánea (TOS) es, hasta la fecha, el único experimento que permite verificar bipótesis y modelos sobre el funcionamiento del babla real, porque en la TOS el hablador llamado «intérprete» tiene que repetir lo que ha comprendido, dándonos con ello acceso experimental no sólo al resultado de la comprensión, sino al proceso de la misma. Nuestros experimentos in vivo sobre las desviaciones deliberadas de la literalidad en interpretación de conferencia (DDL) han demostrado que el intérprete sólo puede interpretar si ha comprendido, es decir, su trabajo no consiste en reproducir las estructuras lingüísticas de una "lengua» reemplazándolas con las estructuras lingüísticas de otra «lengua», sino en reproducir percepciones de usentido" que obtiene, en un primer acto de habla, sin duda mediante las estructuras lingüístico-semánticas, pero, sobre todo, mediante otras muchas cosas que ya no son «lengua». $Y$ reproduce esas otras "estructuras perceptuales" sin duda, también, mediante estructuras lingüístico-semánticas de la otra «lengua», pero también mediante otras muchas cosas.

En una segunda serie de experimentos, en 198 I - interrumpida por falta de fondos-, en colaboración con el Laboratorio de la Comunicación Hablada de la Universidad Católica de Lovaina, hemos podido comenzar a demostrar que el intérprete no percibe palabras que se emiten fuera de todo contexto y que, por otro lado, "oye" palabras que no existen, pero que el percepto de sentido, una vez constituido, proyecta ilusoriamente en la percepción sonora. Lo que demuestra el principio de la "primacía del sentido", es decir, la primacía del percepto como proceso que pilota toda la actividad de la percepción.

Estas series experimentales nos permiten resumir nuestro modelo diciendo que «no percibimos primero la frase y luego la idea, sino al revés; primero percibimos la idea, y porque la percibimos, oímos la frase».

\section{PRESENTACION DEL MODELO EN NOTACION SIMBOLICA}

La notación simbólica que hemos desarrollado tiene por objeto sola y exclusivamente el facilitar la dicción. El modelo del acto social del habla es sumamente complicado. Entran en juegos muchos conceptos y muchas relaciones entre ellos. Nos ha parecido más útil el proceder con una notación simbólica porque se ve más claramente la cosa, es más fácil de memorizar y facilita luego la discusión. Amén de dar mayor precisión a los conceptos.

Así hablamos del acto de habla Do (Discurso de un Orador) o del acto de habla Di (Discurso de un Interlocutor o de un Intérprete). Hablamos en general de un Do y decimos que:

$$
\begin{aligned}
& \text { Do: } \beta_{0}^{K} \rightarrow\left|\Phi_{o} X M^{\lambda} . S m^{\pi_{1}}\right| \Gamma_{o}^{\pi} \rightarrow \Sigma_{o}^{K} \\
& \text { si } \Sigma_{0}=\beta_{0}
\end{aligned}
$$

en que:

Do es el acto de habla en cuestión celebrado en la lengua «o» (Discurso del orador).

$\beta$ es el percepto que el sujeto de la producción (nuestro interlocutor) nos quiere comunicar; está afectado de un subíndice «o» que representa a la vez la lengua "O» y el hecho de que esa actividad social se celebra en el marco del acto social de habla Do, que tiene lugar a tal hora y en tal sitio; y está afectado de un exponente $\mathrm{k}$ que simboliza el sistema estructural de todos los conocimientos previos y compartidos (que están en la memoria permanente de ambos suje- 
tos y, por tanto, en la memoria social colectiva) necesarios para que el sujeto de la producción pueda concebir el percepto $\beta$ que quiere decir, y entre ellos, el conocimiento de la lengua "o».

- simboliza el hecho social de la emisión o enunciación del signostructo (es mejor decir "signostructo" o conjunto organizado de signos, que «frase», porque con frecuencia no se trata de una "frase»).

$\Phi$ simboliza la unidad formal del signostructo, es decir, el signostructo en cuanto percibido como una unidad de forma simbólica, unidad de forma que el sujeto de la producción percibe como tal unidad en el momento de la producción y emisión, y que el sujeto de la comprensión percibe también como una unidad de forma cuanto capta el sentido, es decir, cuanto construye o sintetiza el percepto Sigma; va afectado del subíndice "o» para simbolizar que esa forma ha sido emitida en el marco del acto social Do, y que su contenido o signostructo es una entidad lingüística construida con los signos y reglas de la lengua «o».

$\mathrm{Xm}$ simboliza la estructura de superficie chomskyana, es decir, la estructura gramatical, o estructura fono-morfoléxico-sintáctico-prosodia, y la llamamos «m» para indicar que es una estructura cualquiera, esa que ha sido emitida en esta ocasión, que hubiera podido ser otra, a saber, $\mathrm{Xn}$, o Xp; y Xm va afectada de un exponente $\lambda$ que simboliza la totalidad del sistema estructural de la lengua "o», ausente, pero que gravita sobre el acto de habla y más concretamente sobre los habladores, lo que significa que ese sistema no es un hecho que aparezca en el acto, es decir, que no se actualiza, sino que está ausente $y$, sin embargo, gravita desde su ausencia, ya que los habladores no podrían construir frases sin ese sistema; y aunque lo único empírico y fáctico sea el signostructo emitido, es evidente que éste no podría existir sin el sistema que se revela en la actualización del signostructo, de modo que se puede decir que en cada frase está toda la gramática de una lengua, pero no fácticamente presente.

Sm simboliza y representa la estructura semántica del signostructo, es decir, lo que cada signo, en cuanto significante, significa, y lo que todo el $\mathrm{Xm}$, en cuanto significante, significa; por ejemplo, la $\mathrm{Sm}$ de "good morning" es "buenas mañanas» aunque su sentido en castellano sea «buenos días», o bien la $\mathrm{Sm}$ o masa semántica de "awareness» es "darse cuenta de algo", aunque su sentido en castellano sea, con frecuencia, pero no necesariamente siempre, "conciencia», quedando entendido que esta distinción entre la masa semántica de un signo o de un signostructo y su sentido sólo es perceptible en traducción porque el traductor tiene a diario constantemente la experiencia de que no puede reproducir la masa semántica que en el sistema semántico de una lengua «o» tenga un signo $o$ un signostructo, sino que tiene que reproducir su "sentido", el cual puede ser muy distinto, en un acto de habla concreto, de dicha masa semántica, y quedando entendido que todo signo aislado y todo signostructo aislado (es decir, fuera de un acto de habla concreto) son polisémicos, es decir, tienen varios significados, como, por ejemplo, el signo «administración» puede significar administración del estado (en cuyo caso el significado es "gestión») y administración de un fármaco (en cuyo caso el significado es «dar» o "dación»), o bien, por ejemplo, el signostructo «he comprado el periódico" tiene una masa semántica $\mathrm{Sm}$ compuesto del conjunto $\mathrm{Sm}=\left(s_{1}, s_{2}, s_{3}\right)$, -lo que se aplica a toda $\mathrm{Sm}$ - en el que $S_{1}=$ whe comprado un ejemplar del periódico", y $s_{2}=$ "he comprado la empresa que edita el periódico», $s_{3}=$ «he sobor- 
nado al director del periódicon (García-Landa, 1978), y no sabremos cuál de esos tres significados, o tal vez otro, es el pertinente hasta que ese signostructo no haya sido emitido en un acto real de habla y haya, por tanto, un hablador que le dé «sentido» al comunicar un espacio perceptual Beta; y Sm lleva un exponente $\eta$ (eta) que simboliza y representa el sistema estructural semántico de la lengua "O», ausente de la facticidad empírica del acto de habla, pero, no obstante, que gravita sobre el acto de habla en cuanto que gravita sobre los habladores, y es, en cuanto tal sistema ausente, en todo igual al sistema $\lambda$ de la lengua «o».

$\Gamma$ simboliza y representa la «situación» social del acto de habla, o sea, el acto de habla en cuanto ceremonia social que se celebra en tal sitio y en tal momento, en tales circunstancias, con tales personas, y sus gestos, mímica y expresión en general, y el tal símbolo va afectado de un exponente $\pi$ que simboliza y representa el sistema estructural de las normas sociales (pragma) que afectan la praxis de los actantes o actores del acto y que el sujeto de la comprensión tiene que conocer - aunque su «conocimiento» puede ser inconsciente e incluso inarticulable - y que, en cuanto tal sistema, está ausente de la facticidad empírica del acto de habla, pero, no obstante, gravita sobre el acto de habla, puesto que el sujeto de la comprensión (y el de la percepción) lo tiene que conocer para interpretar correctamente lo que el otro le quiere decir, es decir, para constituir un espacio perceptual Sigma que sea de alguna manera idéntico al espacio perceptual Beta que produce el sujeto de la producción, teniendo dicho sistema estructural todas las características que tienen los otros sistemas ausentes pero gravitantes que aparecen como exponentes y que por eso llamaremos "sistemas exponenciales».

$\leftrightarrow$ simboliza y representa la interacción de la entrada («input») de datos en el sistema cognitivo, $y$, por otro lado, la actividad activa de este sistema tal como se manifiesta en los diversos procesos que pone en marcha, y entre ellos, en la formulación y verificación de hipótesis de percepción, para elaborar dichos datos y producir por síntesis el percepto Sigma, al que se refiere el párrafo siguiente.

$\Sigma$ simboliza y representa el percepto sintetizado por el sujeto de la comprensión (es el «sentido» de los traductores en contraposición a la masa semántica Sm del signostructo) y va afectado de un subíndice «o» para simbolizar su pertenencia al acto de habla Do (y no a otro acto de habla, como, por ejemplo, el Di), y va afectado de un exponente $\mathrm{k}$, que representa el sistema exponencial de todos los conocimientos previos y compartidos con el sujeto de la producción necesarios para que el sujeto de la comprensión puede sintetizar el percepto $\Sigma$.

Finalmente, el modelo tiene que postular la identidad $\Sigma_{o}=\beta_{o}$, es decir, la identidad entre lo que el sujeto de la producción "quiso decir» y lo que el sujeto de la comprensión comprendió. Tiene que existir un mínimo de identidad entre ambos para que se pueda hablar de comunicación, pero el modelo no quiere decir nada más sobre esa identidad.

El modelo de la traducción consiste en decir que el intérprete participa como sujeto de la comprensión en un acto de habla Do, en el cual produce un $\Sigma_{0}=\beta o$, y que, luego, pone en marcha un segundo acto de habla, Di (Discurso del intérprete), en una segunda lengua o lengua «i», y en cuanto sujeto de la producción produce un $\beta i$ que tiene que producir en quien lo escuche un $\Sigma_{\mathrm{i}}$ que tiene que satisfacer a la condición $\Sigma \mathrm{l}=\beta_{\mathrm{o}}$, para que se pueda hablar de traducción, es decir, de reproducción del "sentido", ya que ésta y no otra es la misión del traducir, reproducir espacios perceptuales sigmáticos y no estructuras lingüisticas 
del tipo Xm.Sm. (No presentamos la "fórmula" en notación simbólica de la traducción porque no es esto lo que nos interesa en este trabajo, pero no es dificil de imaginar si el lector tiene en cuenta que Di: $\left.\beta_{l}^{k} \rightarrow\left|\Phi_{l} \mathrm{Xn}_{n}^{\lambda} \mathrm{Sn}^{\eta}\right| \Gamma_{l}^{\pi} \leftrightarrow \Sigma_{1}^{k}\right)$.

\section{TRADUCCION DEL MODELO EN NOTACION SIMBOLICA} A UNA DICCION LITERARIA

El modelo quiere decir que cuando un interlocutor (sujeto de la producción) nos quiere transmitir un espacio perceptual Beta, construye y emite un signostructo o construcción de signos, según reglas, que percibimos como una forma simbólica o unidad formal $\Phi$ en cuanto que percibimos su "sentido» total, es decir, en cuanto constituimos un espacio perceptual Sigma. Y constituimos este espacio perceptual Sigma, no porque entendamos el signostructo (cuya unidad es $\Phi$ ), sino porque, a partir de los datos semánticos que contiene $(\mathrm{Sm})$ y en función de ciertos conocimientos (k) y de ciertos saberes pragmáticos $(\pi)$, constituimos una hipótesis, un primer espacio perceptual Sigma, que verificamos rapidísimamente (según nuestra segunda e interrumpida serie de experimentos en unos cincuenta milisegundos), contrastándola con todos los datos, tanto los lingüístico-semánticos, como los contenidos en $\mathrm{k}$ y $\pi$. Una vez construido el percepto Sigma, podemos constituir definitivamente el contenido lingüístico de la unidad de forma simbólica $\Phi, y$ podemos analizarlo y ver que tiene una estructura de superficie dada (Xm) y una estructura semántica también dada (Sm).

Dicho de otra manera: comprender «el lenguaje» consiste en producir un precepto suprasensorial, "sigmático», en determinadas condiciones definidas por las hipótesis del modelo. La comprensión «del lenguaje» es una especie de percepción, producida por el mismo sistema cognitivo que produce la percepción sensorial, utilizando los mismos aparatos, los mismos mecanismos, los mismos procesos, las mismas actividades $y$, por eso, para comprender esa comprensión hay que empezar por verla como una percepción. Comprender el lenguaje no tiene nada que ver ni con la lingüística ni con la lengua en cuanto sistema de signos. Es un asunto psicológico que sólo la psicología puede explicar. Y sólo la puede explicar si aplica a ese fenómeno la misma metodología que aplica a la percepción sensorial. Sin embargo, la comprensión «del lenguaje» no es sólo una percepción sensorial. Sin dejar de ser una percepciónsensorial es algo más. Es una percepción cultural, simbólica, percepción de masas sensoriales que constituyen el soporte de formas simbólicas con sentido. Decir «forma» es decir "sentido" (en este contexto). $Y$ por eso la percepción suprasensorial sigmática no es sólo psicológica sino que es psicosocial. Porque el percepto sigmático que yo constituyo es el percepto Beta que tú me querías comunicar. No es un mero cuerpo natural sensible, sino otro miembro de la misma especie, otro miembro de mi grupo social quien es la fuente del cuerpo sigmático que tengo que percibir. $Y$ si percibo su percepto Beta con mi percepto Sigma, ambos fenómenos psicológicos, la identidad entre ellos establece una comunidad, una comunión, una puesta en común que no sólo es social, sino que es el concepto mismo de sociedad. Pero, a los efectos de la exploración experimental de todos esos fenómenos, es suficiente con decir que se trata de un asunto psicológico. Tanto más cuanto que sólo la psicología experimental puede tener acceso de momento a esos fenómenos.

Esto es, traducido a una dicción literaria, lo que quieren decir las hipótesis contenidas en nuestro modelo del acto de habla. $Y$ así es como hay que leer la expresión en notación simbólica que hemos presentado más arriba. Esa expresión es sumamente útil para el hombre de ciencia porque le ahorra toda la palabrería literaria resumiendo lo esencial en una serie de simbolos y relaciones entre símbolos fáciles de memorizar si uno se toma la molestia de escribir la «fórmula» diez veces seguidas. 


\section{LOS PRINCIPIOS DEL MODELO}

\section{I. ${ }^{\circ}$ El principio de la diferencia entre lo} semántico (fenómeno linguiistico) y el percepto sigmático (que es un objeto psicológico).

Esta distinción es capital. Ya hemos hablado de ella pero conviene insistir porque no ha sido formulada en ninguna parte y constituye una sugerencia tan nueva que pudiera resultar dificil de comprender. Hasta ahora, las teorías o modelos de la "percepción del lenguaje» dan por supuesto que la estructura semántica de la frase es unívoca y penetra, en cuanto tal, en el sistema cognitivo. La experiencia práctica de la traducción y los experimentos realizados por nosotros (García-Landa, 1978), demuestran que eso no es, en absoluto, así sino que la estructura semántica de una frase, su masa semántica $\mathrm{Sm}$, pertenece al sistema semántico de la lengua (al diccionario) y no es eso lo que se percibe ni lo que entra en el sistema cognitivo en cuanto a lo percibido. Lo que entra en el percepto es el «sentido» que el sujeto de la producción quiere comunicar (quiere decir) - su percepto Beta-utilizando esa masa semántica para sus fines, unos fines que, en relación con la generalidad y objetividad sociales del sistema semántico, se pueden calificar de «subjetivos». Para demostrar la diferencia entre lo semántico y lo sigmático no hay más que aludir al hecho universal de que, en toda lengua, los significantes son siempre polisémicos cuando están aislados (o sea, cuando están en el diccionario, ya sea éste concebido como un libro o como una memoria permanente en los sujetos hablantes) y, por tanto, ambiguos cuando aparecen en un acto de habla real y concreto. La polisemia de la lengua conduce a la ambigüedad del habla (García Yebra, I 98I). Piénsese en la polisemia, y consiguiente ambigüedad, de un signo como «subjetivo, subjetividad», con los que cada orador o escritor quiere decir una cosa distinta. Piénsese en la polisemia, y consiguiente ambigüedad, del signostructo «he comprado el periódico».

Esta distinción entre lo semántico y lo sigmático es capital y recorre nuestro modelo de arriba a abajo. Los principios que enumeramos a continuación son un corolario de esa distinción.

2. ${ }^{\circ}$ El percepto Sigma no es el resultado de la mera entrada de los datos lingüísticos $\mathrm{Xm}$ ni de la masa semántica Sm en el sistema cognitivo - como pretenden las teorías o modelos llamados «lingüísticos»-, sino el resultado de la interacción de Xm.Sm con los conocimientos $x$ y los saberes pragmáticos $\pi$ (entre los que figuran los datos proporcionados por la expresión, mímica, gestos del interlocutor). Está claro que si no hubiera ninguna diferencia entre lo semántico y lo sigmático, el modelo de la "percepción del lenguaje" se limitaría a decir que percibir una frase es percibir su estructura semántica. Dicho sea de paso, nuestro concepto de Sm o masa semántica es distinto del concepto chomskyano de «deep structure» y la única manera de relacionarlos sería decir que lo que Chomsky llama «deep structure» se desdobla en nuestro modelo en dos cosas: la masa semántica Sm y el percepto Sigma.

3..$^{\circ}$ El principio de las expectativas. Entre esos conocimientos, de tipo $x \circ \pi$, hay que incluir las expectativas del sujeto de la comprensión que sabe de antemano mucho sobre lo que su interlocutor (el sujeto de la producción) le va a decir. Basta descifrar las primeras palabras de una frase para saber de qué nos va a hablar el otro. A veces es suficiente entrar en una situación social dada para saber lo que nos van a decir. Por ejemplo, al entrar en un restaurante sabemos de antemano lo que nos va a decir el jefe de sala y lo que le vamos a contestar. Y esto se aplica a la mayoría de las situaciones sociales que vivimos a diario, incluyendo las situaciones específicamente intelectuales. En otras palabras, es muy difícil encontrar ideas originales, no sólo porque hay pocas, sino porque si las encontráramos no las comprenderíamos, ya que no estamos preparados. Este principio de las expectativas comprende el principio de los conocimientos compartidos o consabidos. Seria imposible com- 
prendernos si no compartiéramos -en una memoria permanente que es social e individual al mismo tiempo, como se ve en la "lengua»- un fondo de conocimientos comunes. Tres corolarios: a) hay que conocer el "contexto" conceptual en el que se sitúa el espacio Beta que se trata de comprender y ese espacio conceptual "a priori» no es comunicado por lo dicho, sino activado en la memoria permanente; b) la noción de conocimientos compartidos o consabidos se parece mucho a lo que se conoce en psicolingüística con el nombre de "given/new contract», según el cual todo interlocutor supone o da por supuesto que su interlocutor posee una serie de conocimientos ("given») en los que vienen a colocarse las nuevas informaciones («new») que quiere comunicar y esto es un contrato social tácito entre interlocutores, y c) entre esos conocimientos figura el saber pragmático (no verbalizable) de las normas sociales que rigen el acto social del habla.

\section{EL ASPECTO DINAMICO DEL MODELO: LA SINTESIS DEL SENTIDO}

Hay que imaginarse el acto social del habla como una fábrica en la que diversos factores concurren para la producción de un objeto llamado «espacio perceptual Sigman. Estos factores son: el significante (Xm), el significado (Sm), la forma simbólica (o), los conocimientos $x$ y los saberes $\pi$. Todos estos factores fusionan en un momento dado en el proceso perceptual de la comprensión sigmática para constituir una unidad cognitivo-perceptual $x$. Es el momento de la sintesis sigmática. Este percepto, una vez sintetizado, pilota el proceso total de la comprensión. Clark y Clark (1977) expresan esta idea diciendo: According to Lieberman, much of the speech people bear is ultimately what they themselves have synthesized and not what the speaker has produced. The reason this speech is clear is because it is self-generated. In this sense the clarity of speech is normally an illusion. (Según Lieberman, buena parte del habla que la gente oye es, en último término, lo que ellos mismos producen por síntesis y no lo que el interlocutor ha producido. La razón de que les parezca claro eso que creen que oyen es porque son ellos mismos quienes lo han producido. En este sentido, la claridad en el hablar es normalmente una ilusión.)

\section{TOTALIDAD DEL ESPACIO SIGMATICO Y SUS MOMENTOS: EL PRINCIPIO DE LA PRIMACIA DEL SENTIDO}

Salvo, tal vez, al principio de una conversación inusitada, se sintetiza primero el espacio perceptual Sigma y sólo después se "oyen" las "palabras" que permanecen en la memoria auditiva (unos 250 milisegundos) esperando que el percepto Sigma se produzca. Una vez que dicho percepto se ha producido, su contenido se retroproyecta en las "palabras", que en ese momento "se cargan de sentido" o "cobran sentido". Y así se crea la ilusión de que tenían sentido de antemano y de que comprender lo que alguien quiere decir es comprender la frase, y eso es todo. Cuando, en realidad, la "frase sólo existe después de que se ha captado el sentido que el interlocutor quiere decir (después de la sintesis del espacio Sigma). Esto puede sorprender (deberia sorprender y chocar) a los que creen a pies juntillas en los modelos lineales de la comprensión, según los cuales se perciben primero los fonemas con los cuales se construyen las sílabas con las cuales se construyen los morfemas con los cuales se construyen las palabras con los cualesse construye la sintaxis, con todo lo cual se construye la "deep structure», y eso es todo. Pollack y Pickett (1964) han grabado conversaciones corrientes y lecturas de textos y han aislado las partes de la cinta que sólo contenían palabras aisladas $y$ se las han hecho escuchar a otros sujetos. Resultado: «palabras aisladas como éstas fueron identificadas correctamente sólo en el 47 por 100 de los casos, porcentaje sorprendentemente bajo", como dicen Clark y Clark (1977), quienes añaden: «Aunque Pollack y Pickett pre- 
sentaron al principio sólo palabras aisladas, fueron añadiendo luego segmentos cada vez mayores de la cinta detrás de cada palabra. A medida que iba aumentando el segmento de habla, los sujetos iban adivinando mejor y mejor. Podían utilizar más y más del contorno acústico, sintáctico, prosódico en el que esa palabra estaba colocada. La impresión subjetiva dominante, sin embargo, no ve en ello un aumento progresivo de la inteligibilidad. Al revés, se diria que la palabra permanece ininteligible hasta que se llega a un cierto punto, en el cual (de pronto) se comprende con toda claridad.» El momento o punto en que de pronto se comprende - añadimos nosotros- es el momento de la síntesis del espacio perceptual Sigma. Obsérvese que tal como se describe ese experimento, se revela que la hipótesis implícita es que basta oír las estructuras lingüísticas y nada más. Nuestra interpretación del mismo experimento --partiendo de una serie distinta de hipótesis (las contenidas en nuestro modelo)tendria en cuenta otros muchos ingredientes. Por ejemplo, el ingrediente del tema. Los sujetos no conocían el tema, no habían oído el «discurso» original, sólo se enfrentaban con palabras aisladas. El 47 por 100 de los casos no percibían nada, mero ruido fónico. A medida que se añadía más cinta, no sólo se añadía más material verbal, sino que en un momento dado el tema tenía que ser visible.

\section{EL EFECTO DEL TELEX}

He aquí un proyecto de experimento que nunca hemos realizado, pero con el que hemos jugado con frecuencia. Se trata de observar la máquina de escribir del télex cuando está funcionando. Como se sabe, es una máquina operada a distancia, no hay nadie delante, el sujeto del experimento puede colocarse delante y leer con toda comodidad las letras según las va tecleando la máquina. Las primeras letras no producen ninguna reacción, pero a partir de un momento dado se sabe de antemano cómo va a terminar la frase. No se trata de un proceso estocástico tipo
Markov, sino de algo todavía más complicado. En un momento dado se capta el tema y se puede adelantar el résto de la noticia.

\section{LOS MOMENTOS DE LA TOTALIDAD Y LA TOTALIDAD REAL TAL COMO SE VIVE}

Además de la subjetividad de los sujetos hablantes - «subjetividad» en cuanto opuesta a la «objetividad» del sistema $\lambda \eta$ - hemos hablado de otros factores o momentos de la producción del percepto Sigma, como son el signostructo en su doble vertiente de estructura gramatical $\mathrm{Xm}$ y de masa semántica $\mathrm{Sm}$, asi como la situación Gamma y su sistema exponencial $\pi$ y el sistema exponencial $\pi$ (o universo sénsico en cuanto diferente del universo semántico). No hay que pensar que todos esos "fenómenos" sean entidades o cosas u objetos dotados de una existencia propia. No son sino momentos de la totalidad de la interacción social que es el acto de hablar. Ni siquiera tienen la efímera sustancialidad de esas partículas subatómicas que duran «el tiempo de un suspiro", pero, por muy efímeras que sean, son "cosas» con trayectoria espaciotemporal y todo.

Desde el punto de vista de la praxis traductora, nos vemos obligados a hacer una diferencia radical entre lo semántico o lo sigmático o «sentido» porque, desde hace miles de años, los traductores repiten que no traducen palabras sino el sentido. Nuestro modelo separa así claramente la estructura gramatical $\mathrm{Xm}$ y la estructura semántica $\mathrm{Sm}$ del percepto Sigma (que consiste en su identidad con el percepto Beta, por lo que basta citar aquél para mentar éste) para mostrar cómo en la traducción el "sentido" es diferente de las "palabras», lo que, de paso, le viene muy bien a la psicología experimental, que puede distinguir así entre el percepto percibido y los medios de transmisión y constitución del mismo. En la realidad del habla viva, tal como se vive, todos esos momentos forman un 
todo que se percibe como un todo. Los gramáticos y los lingüistas matan esa totalidad viva para hacerle la autopsia y acumular un corpus de $\mathrm{Xm}$ y de $\mathrm{Sm}$, de los que extraen el sistema exponencial $\lambda \eta$ (que es lo que, desde Sausurre, llamamos la «lengua»). Los teóricos de la traducción - y, espero, los psicólogos experimentales - matan esa misma totalidad viva para extraer el sentido o percepto Sigma, separándolo de los objetos o entidades «lingüísticos».

En la espontaneidad del habla real o viva vivimos la totalidad del percepto Sigma sin separarlo de las palabras. Nuestro modelo postula que, una vez constituido el percepto Sigma, éste se retroproyecta en el signostructo $\Phi_{\mathrm{o}} \mathrm{Xm} . \mathrm{Sm}$, de tal modo que este signostructo se nos aparece como cargado de sentido y como si el sentido estuviera en él. Esto es aún más patente en la lectura, puesto que el texto, en cuanto escrito, no desaparece y la vista puede volver a la página una y otra vez... después de haber comprendido. De ahí que, en la vida cotidiana, no hagamos esas finas y sofisticadas distinciones entre lo que es de la «lengua» y lo que es del "percepto" y llamemos a todo lengua. O lengúaje, con la misma imprecisión. Esta es la impresión que tenemos en la cotidianeidad. Se diría que la cotidianeidad tiene su verdad, que es muy distinta de la verdad que encuentra la ciencia. $Y$ se diría también que en las ciencias, sobre todo en las sociales, no sólo hay que explicar científicamente los fenómenos, sino también explicar por qué se ven como se ven en la cotidianeidad. Por ejemplo, en la visión cotidiana el sol es una canica de fuego que se levanta por el levante, se alza al cielo y luego baja y se pone por el poniente, verdad cotidiana que salta a la vista. La ciencia ha descubierto otra verdad distinta, pero ésta no cancela la verdad "social» cotidiana. Quedando entendido que las verdades cotidianas son, ni más ni menos, los prejuicios vigentes en una sociedad..., los cuales desempeñan, en cuanto "precomprensión» o "foreunderstanding", un papel crucial en toda comprensión sigmática.

\section{OBSER VACIONES DE METODO Y COMPRENSION DE ESTE TRABAJO}

El presente trabajo tiene por objeto presentar una serie de hipótesis como marco hipotético de un programa de investigación psicoexperimental de la llamada «percepción del lenguaje». Las hipótesis se presentan en forma de postulaciones, es decir, de afirmaciones gratuitas en el sentido de que no se pretende demostrar su verdad, ya que partimos de la base de que la única demostración de ese tipo es la que pueda proporcionar el experimento, aunque, en rigor, sería suficiente que el experimento mostrara la capacidad de ese marco hipotético para, no sólo explicar, sino, sobre todo, explorar el campo así descubrible y cartografiable.

No se nos puede pedir, pues, que demostremos la verdad de tales postulaciones hipotéticas. Lo que sí se nos podría pedir es que al menos mostremos su alcance, su contenido, su sentido, su valor; es decir, que las hagamos comprensibles y plausibles para el lector, nuestro interlocutor en este acto de habla escrita. Confesamos que, deliberadamente, no hemos intentado esto tampoco, porque alargaría demasiado el texto, salvo en el caso de la hipótesis que dice que lo lingüístico-semántico es distinto de lo sigmático (del percepto sintetizado), y en el caso de la hipótesis que dice que para comprender lo que un interlocutor nos quiere decir - por oral o por escrito- hay que saber pragmáticamente (es decir, más o menos conscientemente) las normas sociales que rigen el acto de habla. En ambos casos el intento ha consistido en dar algunos ejemplos o en mencionar la polisemia constitutiva de toda lengua y la consiguiente ambigüedad de todo hablar. Esta ascesis se debe a que estamos convencidos de que sólo la experimentación (o la praxis de la traducción) puede revelar el contenido, alcance, sentido y valor de esas hipótesis.

Sin embargo, para que el lector tenga ocasión de hacerse una vaga idea del 
alcance del marco hipotético en su conjunto, en cuanto teoría capaz de descubrir, explorar y explicar el fenómeno de la comprensión del habla, vamos a intentar un pequeño «experimento» que, dado que estamos aquí en un acto de habla escrita en el que la comprensión se presenta como «lectura", será un experimento de lectura. La diferencia entre "lengua» (que comprende la semántica o sistema de significados) y «habla» que postulan nuestras hipótesis, se transforma en la lectura en diferencia entre deletreo y verdadera comprensión. Vamos a presentar al lector un "texto" en el que hemos trastrocado el orden de las letras en cada palabra para conseguir que el lector separe claramente el momento del deletreo del momento de la comprensión:

OLS ESRIPITUS FICIALESPERSU CANBUS OSL GEORINES ED TAES SICRIS NE AL LUVOCIONRE CESAFRAN, QEU ON OZIH ONIS LACARSA A AL FICIERUPES, DUANCO NE READALID SE HERENTEIN NE LE TIACRISNISMO DEDSE PRESIEM, DIDIVIDO NETRE ANU RALMO MACATIDOG TAPUESIM ROP LE DREPA Y ANU SIFICATEMA ANARTAQUIS DANACAREN NE LE JOHI.

En el difícil deletreo el lector realiza una operación puramente lingǘstica de reconocimiento de palabras, de signos, retrasando así el momento de la sintesis del percepto Sigma, en el que surge la «idea», cuya comprensión pone en juego, no los conocimientos de la lengua, sino los conocimientos culturales de una determinada religión tradicional en ciertas regiones del globo y totalmente desconocida en otras, así como los conocimientos de las teorías de Freud sobre el conflicto edipiano entre el padre y el hijo. Un japonés corriente y moliente, que seguramente no tiene en su bagaje cultural tradicional ni la más ligera idea de dogmas, metafísicas, trinidades divinas o edipianas, podrá tal vez descifrar el deletreo de los signos si conoce el castellano, pero, epodrá "comprender» la idea que el autor del texto intenta comunicar al lector? El texto ha sido tomado de Raymond
Abellio, Ma derniere mémoire, un faubourg de Toulouse, Paris, Gallimard, 1971, págs. 54-55, traducido al castellano y criptado por nosotros.

¿Qué piensa ahora el lector de las teorías que pretenden que para comprender (o percibir) una frase basta con conocer la «lengua»?

Para terminar, una cita del texto que nos ha inspirado la idea de este experimento:

«Por su lado, las investigaciones consagradas a la psicolingüística de la comprensión distinguen en la lectura entre el "acto léxico" y el "acto escriptural": el niño que va a la escuela aprende a leer al mismo tiempo que aprende a descifrar, pero la primera actividad no está determinada por la segunda. Dicho con otras palabras, reconocer los signos, o deletrear, no es lo mismo que leer. Leer, es decir, que el lector pueda abordar el texto con estrategias de interrogación semántica depende de una memoria cultural, depositada por los intercambios orales y relativa a la audición. El texto, una vez descifrado, se constituye solamente en función de ciertas expectativas de sentido que el mismo texto corrige y afina, como si la construcción de significaciones hipotéticas fuera un bloque de mármol que poco a poco viniera a esculpir, desplazar, precisar, verificar el descifrado de los materiales gráficos para dar lugar a formas de lectura, como si el grafismo fuera trabajando en una anticipación de sentido. La lectura obedece a la misma ley que el experimento científico: es tanto más rica cuanto más numerosas son las expectativas e hipótesis a priori.» (Certeau, I98 I.)

Obsérvese que el autor de este texto emplea todavía las expresiones «semántica» y "significación» en el marco hipotético que regía antes de que nosotros formuláramos el nuestro. Traducido a nuestro «vocabulario», «leer es abordar el texto con estrategias de interrogación sigmática...» y «... como si la construcción de perceptos sigmáticos hipotéticos...». En las demás partes prevalece el uso tradicional de usentido", que representa 
una corriente cultural tradicional en completa contradicción con las hipótesis de las lingüisticas contemporáneas sin que nadie se haya tomado la molestia de examinar con lupa esa contradicción hasta que la praxis de la traducción nos llevó a nosotros a hacerlo.

Por otro lado, lo que este texto llama "acto léxico» es, más o menos, lo mismo que lo que nosotros llamamos upercepción de la frase» (o del signostructo), mientras que lo que llamamos «percepto Sigma», resultado de la comprensión del habla, es lo que este texto francés, siguiendo las modas intelectuales parisinas, más literarias que científicas, llama «acto escriptural». No hay que olvidar que, según nuestras hipótesis, no está dicho que el «acto léxico» preceda siempre de manera separable en la cadena temporal al «acto escriptural». Sin olvidar que las condiciones y circunstancias de la comprensión en el acto de habla oral son muy diferentes de las que rigen en el acto de habla escrita. (La escucha es muy diferente de la lectura..., pero la escucha es el fenómeno primario.)

\section{UN PROGRAMA DE INVESTIGACIONES}

El modelo de la comprensión del lenguaje que hemos presentado contiene una serie de hipótesis verificables mediante una serie de experimentos de Tos. Se trata, como hemos dicho, de un modelo de comprensión del habla real, no de la mera percepción de palabras o frases aisladas. Estos últimos modelos no pueden explicar el fenómeno, ya que la percepción de las palabras y de las frases dependen de la constitución del percepto total Sigma. Nuestro modelo parte de una equiparación de la comprensión a la percepción sensorial para postular la existencia del objeto de la comprensión como una entidad psicológica (no lingüística) distinta del medio lingüístico que se emplea para comunicar el percepto Beta, así como en la percepción sensorial se distingue el medio (luz, sonido) del objeto de la percepción, que es un cuerpo sensible distinto.

\section{Referencias}

Benveniste, 1974 (Emile Benveniste): «La forme et le sens dans le langage», en Problemes de linguistique générale. Paris, Gallimard, I974.

Ci.ARK y Ci.ArK, 1977 (Clark, H. H., y Clark, E. V.): Psychology and Language, An Introduction to Psycholinguistics. Nueva York, H. B. Jovanovitch, 1977.

DoDD y WhITE, Jr., 1980 (Dodd, D., y White, R., Jr.): Cognition, Mental Structures and Proceses, Allyn y Bacon, 1980 .

García LANDA, 1978 (García Landa, M.): “Les déviations délibérées de la littéralité en interprétation de conférence», tesis de doctorado presentada en là Universidad de la Sorbona Nueva (París III) en junio de 1978 , no publicada; ejemplares mimeografiados en la Biblioteca Nacional de Paris, así como en la ESIT de París III y en la ETI de la Universidad de Ottawa.

GARcí YebrA, 198 I (Garcia Yebra, Valentín): "Polisemia, ambigüedad y traducción», en Logos Semantikos, Studia Linguistica in Honorem Eugenio Coseriu, vol. III, I981, Gredos, Walter de Gruyter.

García Yebra, 1982 (Garcia Yebra, Valentín): Teoria y práctica de la traducción. Gredos. Madrid, 1982.

KeLLY, 1979 (Kelly, L. G.): The True Interpreter. Oxford, Blackwell, 1979.

KOLLER, 1979 (Koller, W.): Einfuerung in die Uebersetzungswissenscbaft. Heidelberg, Quelle und Meyer, 1979. 